\title{
FIR MAGNETOOPTICAL MEASUREMENTS ON MOCVD GROWN InAs
}

\author{
T. ANDrearczyk, K. Karpierz, R. Bożek, R. Stegrniewski \\ AND M. GRYNBERG
}

Institute of Experimental Physics, Warsaw University, Hoża 69, 00-681 Warsaw, Poland

In this paper we report results of magnetooptical measurements done on standard InAs MOCVD layers grown on GaAs. Extremely narrow lines (half-widths of the order of $20 \mathrm{mT}$ ) - narrower than found by other authors in high quality MBE InAs epilayers on $\mathrm{GaAs}$ - as well as the lines of typical half-widths have been found both in the photoconductivity spectra and in the transmission spectra. A detailed comparison with the theoretical dependence of shallow donor and Landau level energies on magnetic field leads to the conclusion that they originate from cyclotron resonance and impurity-shifted cyclotron resonance transitions in that material.

PACS numbers: 72.40.+w, 71.55.-i, 78.30.-m

\section{Introduction}

It is known that standard transport measurements on narrow-gap semiconductor layers may give misleading results because of the existence of a space charge layer at the surface or at the interface. The results of Hall measurements done on thin layers might be especially difficult to interpret, because of unknown pattern of the current flow. Therefore the methods, which involve more local interactions with electrons in such semiconductors, give more precise information about the electronic properties of the sample. The intraband and/or shallow donors transitions in many semiconductors give much more "local" insight into the properties of the investigated material [2].

InAs layers and dots have attracted the interest of many researchers. Although in general there is little known about shallow donors in that material, there are groups of researchers who did observe intra-shallow-donor transitions in MBE-grown thin InAs layers on GaAs (see, e.g., Ref. [1] and references therein), as well as cyclotron resonance (CR). However the observed spectra [1] show surprisingly narrow lines of $\mathrm{CR}$, which cannot be compared with the observed values of the carrier mobility obtained from Hall and conductivity measurements in the same samples. The authors of Ref. [1] believe that the extremely narrow lines found in the spectra originate from cyclotron resonance and impurity shifted cyclotron resonance. Unambiguous interpretation of the half-widths of these lines is, however, still under discussion. 


\section{Experiment}

MOCVD InAs samples have been grown on SI GaAs substrates. The sample thickness was of the order of $3 \mu \mathrm{m}$. Indium contacts were attached to the samples. Three different samples from the same wafer were investigated. The typical dimensions of the samples were $5 \times 5 \mathrm{~mm}^{2}$.

The experimental set-up enabled simultaneous measurements of photoconductivity (PC) and transmission (T) spectra for the same sample. All measurements have been done at the LHe temperatures. The samples were placed in a variable temperature cryostat in magnetic fields up to $9 \mathrm{~T}$. Unpolarized, chopped far infrared (FIR) light from a $\mathrm{CO}_{2}$-pumped molecular FIR laser has been used $\left(\lambda_{\text {FIR }}=70.5 \mu \mathrm{m}, 96.5 \mu \mathrm{m}\right.$ and $\left.118.8 \mu \mathrm{m}\right)$. The transmission spectra were measured using a home made, highly sensitive bolometer fabricated from an Allen-Bradley resistor. This bolometer was placed a few $\mathrm{cm}$ behind the sample. The signals were measured using the lock-in technique.

\section{Results and discussion}

Standard van der Pauw measurements of electron concentration $n$ and mobility $\mu$ were carried out. The values of $\mu=9000 \mathrm{~cm}^{2} /(\mathrm{V} \mathrm{s})$ and $n=1.6 \times 10^{16} \mathrm{~cm}^{-3}$

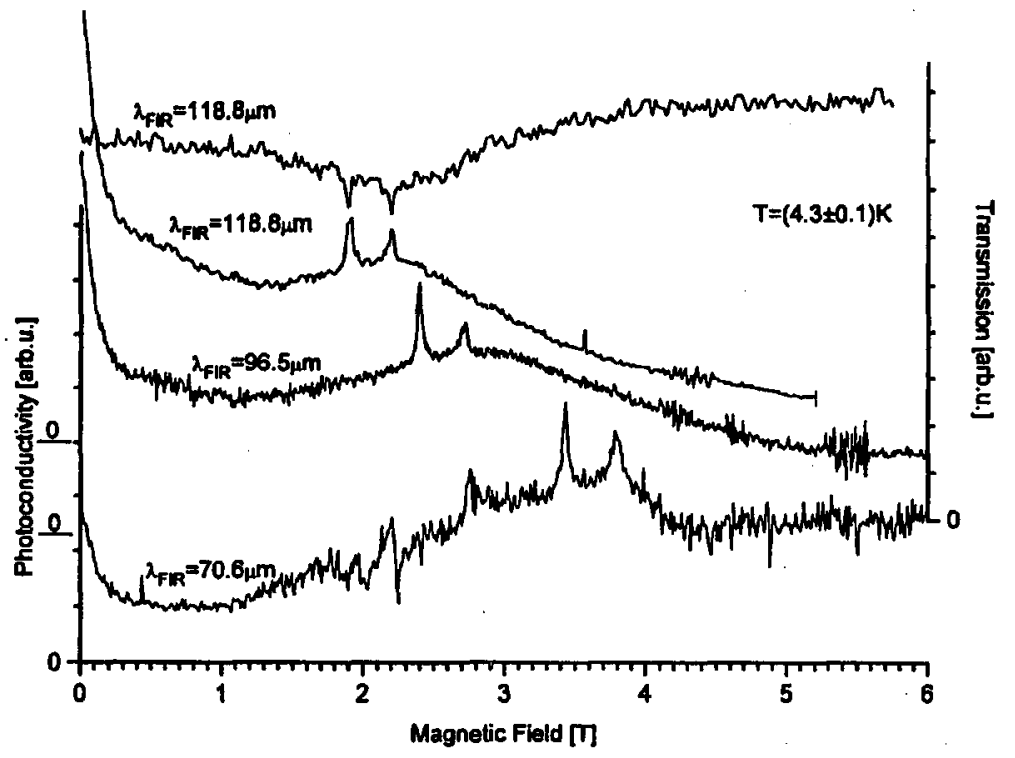

Fig. 1. Photoconductivity and transmission spectra for InAs MOCVD layer. The upper curve shows the transmission spectrum while the three spectra below correspond to the photoconductivity (PC) spectra. The relevant wavelengths of FIR light are marked at each curve. All PC spectra are normalized assuming the same signal level for the highest applied magnetic fields, and are then shifted vertically for clarity. The appropriate "zero levels" are marked on the left axis. 


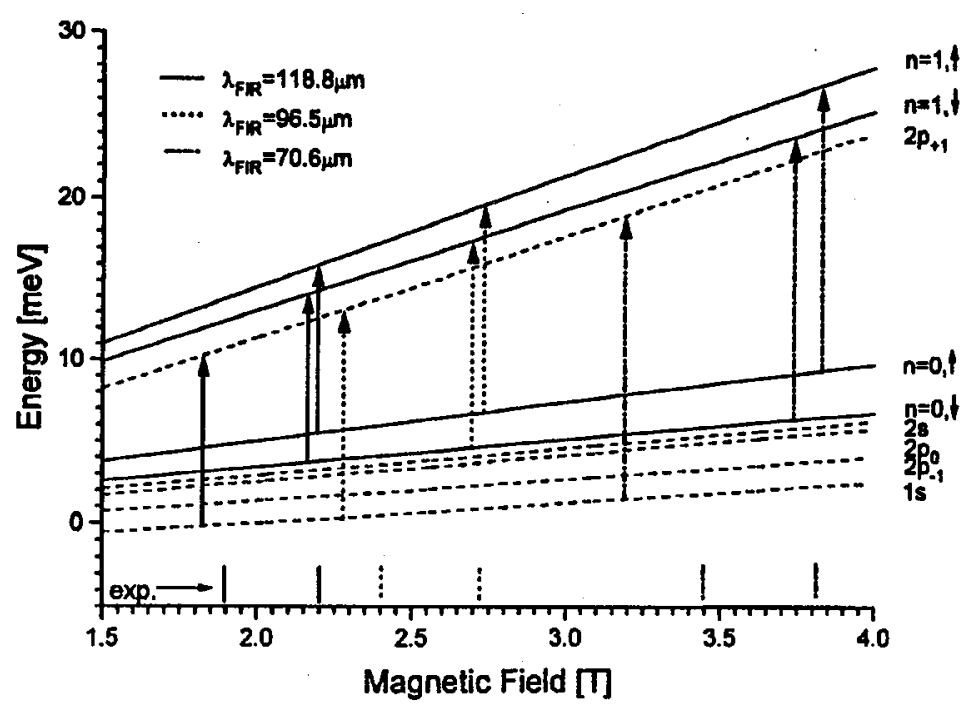

Fig. 2. Theoretical dependence of shallow donor states and Landau levels in InAs versus magnetic field. Solid lines represent the energies of Landau levels calculated from the Pidgeon-Brown [3] model taking into account the nonparabolicity of the conduction band in InAs. Dashed lines represent the energy levels of shallow donor states. The calculations of the hydrogen energy levels in strong magnetic fields have been done by Rosner et al. [4]. The renormalization of the effective mass (assumed value for InAs is $m^{*}=0.023 m_{\mathrm{e}}$ ) and dielectric constant (assumed value for InAs is $\varepsilon_{0}^{*}=15.15$ ) has been done. Vertical arrows represent transitions for the energy equal to the relevant value of FIR photon energy, while the vertical lines below correspond to the experimentally observed values of the peak maxima. The zero of the energy scale corresponds to the energy of the bottom of the InAs conduction band.

for $T=300 \mathrm{~K}$, as well as $\mu=19000 \mathrm{~cm}^{2} /(\mathrm{V} \mathrm{s})$ and $n=0.9 \times 10^{16} \mathrm{~cm}^{-3}$ for $T=77 \mathrm{~K}$ have been obtained.

Figure 1 shows typical spectra obtained in our experiments. We did not notice any change of these spectra from one sample to another. In the spectra one can see two pronounced, narrow peaks. Additionally, as was the case with MBE samples [1], for the MOCVD samples a broad structure on transmission and photoconductivity spectra is also present. It is clear that the broad structure, together with the two narrow peaks, is shifting towards higher magnetic fields with increasing energy of the FIR photons. The same sequence of peaks is also found in the transmission spectra presented in Fig. 1. The detailed comparison of the observed peak positions with the theoretical energy dependence is presented in Fig. 2. It is clearly seen that the positions of the broad peak and the narrow peak in higher magnetic field are exactly the same, and correspond closely to the theoretical positions of the CR transitions. Positions of the impurity-shifted cyclotron resonance fit better for the low field transitions, while the discrepancy in higher magnetic field is well above the experimental accuracy. However, the theoretical curves for the donor levels (dashed) were calculated without taking 


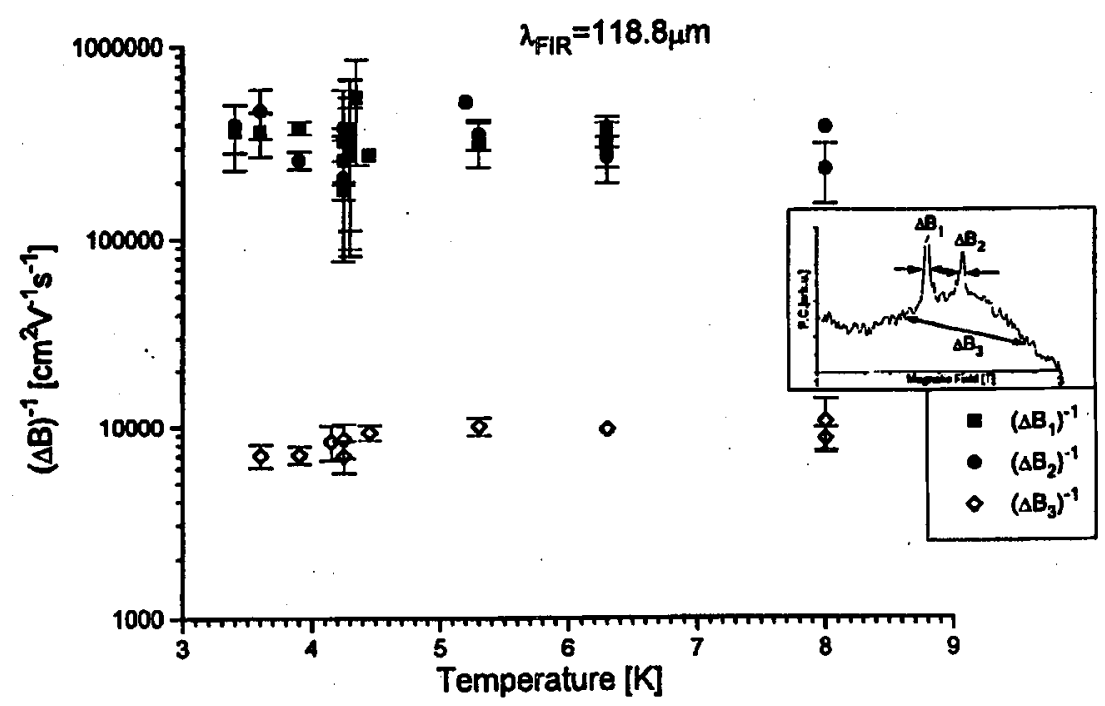

Fig. 3. Temperature dependence of the observed peak half-widths in InAs MOCVD layers for the illumination of FIR light with the wavelength of $118.8 \mu \mathrm{m}$. The inset shows schematically the method of determining the half-widths of the peaks.

into account the spin splitting of the Landau levels and the nonparabolicity of the conduction band.

An additional interesting observable feature is the half-width of the measured narrow CR peak. Figure 3 shows the temperature dependence for all the observed peak half-widths. Surprisingly, the high value of the mobility corresponding to the half-width obtained by the formula

$$
\mu=\frac{1}{\Delta B}
$$

does not have direct correspondence to the observed value of Hall mobility in the investigated samples. Comparing the Hall mobility values with the inverse half-widths of the peaks, it is reasonable to assume that the broad structure is related to the same behavior of electrons as that shown in the transport measurements. Wang et al. [1] attribute this structure to the presence of an interface layer in the samples.

One possible approach to the understanding of the observed narrow peak half-widths is to take into account the potential fluctuations in the investigated layers. It is known that the existence of an interface, and the resulting formation of lattice defects, generates potential fluctuations [2]. In that case one can expect the formation of the regions with locally high quality material. The magnetic lengths are of the order of $200 \AA$ and $120 \AA$ in the magnetic fields $2 \mathrm{~T}$ and $4 \mathrm{~T}$, respectively, and the Bohr radius for shallow donors in InAs is of the order of $350 \AA$. These values give the estimation of the dimensions of the local regions of high quality material, where electrons are not subjected to significantly higher scattering. Comparing the oscillator strengths originating from the broad and narrow CR lines, one can 
estimate that less than $1 \%$ of electrons populates regions with the high quality materials. Therefore the majority of electrons which contribute to the conductivity do originate from the regions, whose properties correspond to those given by the transport (Hall) data.

\section{Acknowledgments}

This work has been partially supported by the Committee for Scientific Research through the grant No. 7 T08A 00209.

\section{References}

[1] P.D. Wang, S.N. Holmes, Tan Le, R.A. Stradling, I.T. Fergusson, A.G. Oliveira, Semicond. Sci. Technol. 7, 767 (1992).

[2] K. Karpierz, J. Łusakowski, M.L. Sadowski, M. Grynberg, Physica B 184, 403 (1993).

[3] C.R. Pidgeon, R.N. Brown, Phys. Rev. 146, 575 (1966); W. Leung, L. Liu, Phys. Rev. B 8, 3811 (1973).

[4] W. Rosner, G. Wunner, H. Herold, H. Ruder, J. Phys. B, At. Mol. Phys. 17, 29 (1984). 